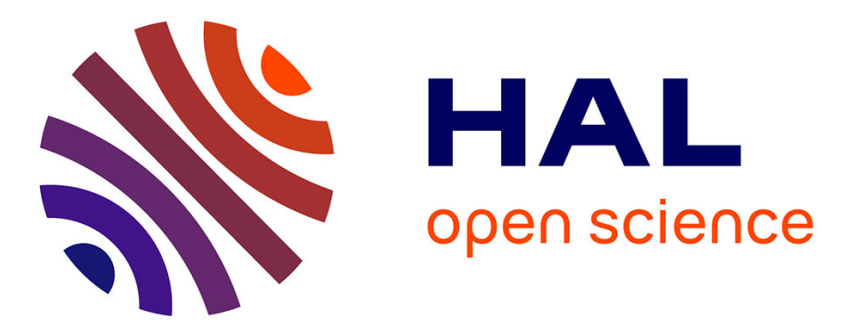

\title{
Le règlement de la lecture des Amadis de Gaule à Lyon : quelques épîtres de Gabriel Chappuys, traducteur
}

\author{
Christine de Buzon
}

\section{To cite this version:}

Christine de Buzon. Le règlement de la lecture des Amadis de Gaule à Lyon: quelques épîtres de Gabriel Chappuys, traducteur. Réforme, Humanisme, Renaissance, 2010, 71, pp.125-149. 10.3406/rhren.2011.3120 . hal-00709014

\section{HAL Id: hal-00709014 \\ https://hal-unilim.archives-ouvertes.fr/hal-00709014}

Submitted on 21 Sep 2018

HAL is a multi-disciplinary open access archive for the deposit and dissemination of scientific research documents, whether they are published or not. The documents may come from teaching and research institutions in France or abroad, or from public or private research centers.
L'archive ouverte pluridisciplinaire HAL, est destinée au dépôt et à la diffusion de documents scientifiques de niveau recherche, publiés ou non, émanant des établissements d'enseignement et de recherche français ou étrangers, des laboratoires publics ou privés. 


\section{Le règlement de la lecture des Amadis de Gaule à Lyon: quelques épîtres de Gabriel Chappuys, traducteur}

Pendant soixante-quinze ans, l'entreprise éditoriale des Amadis a requis une énergie considérable dont il est peu d'exemples dans le domaine romanesque. Plusieurs générations de lecteurs y ont trouvé de l'intérêt et ont soutenu les efforts des traducteurs et des libraires. Amadis de Gaule est publié en français de 1540 à 1615 à Paris, Anvers et Lyon ${ }^{292}$. Certes, tout a commencé à Paris (Denis Janot, in-folio) et c'est dans la capitale que paraissent aussi les trois derniers livres ${ }^{293}$ en 1615. La qualité de la traduction de Nicolas Herberay des Essarts assura, comme on sait, le succès considérable des premiers livres à la cour, à la ville et bien au-delà ${ }^{294}$. Anvers (après 1560, in- $8^{\circ}$ ) et Lyon (après 1575, in-16) ont contribué à ce succès en réimprimant les traductions dans un format de plus en plus plus modeste ${ }^{295}$. Lyon reprend d'abord les livres parisiens (Livres I-XIV) puis se signale par une initiative importante: l'augmentation de la collection par la traduction de livres nouveaux, confiée essentiellement à Gabriel Chappuys, Tourangeau (Livres XV-XXI). Autres initiatives lyonnaises: Huguetan augmente le Thresor (Paris, Groulleau, 1559) en 1571 avant les traductions lyonnaises puis en 1582 avec le Thresor de tous les livres d'Amadis (Livres I-XXI) juste après cette série de traductions ${ }^{296}$. Ce dernier Thresor est reproduit

292. Citons aussi Poitiers et Chambéry. Le Thresor des Amadis - c'est-à-dire un « florilège rhétorique » selon l'expression de M. Bideaux - paraît à Paris et Poitiers en 1559; Guillaume Aubert de Poitiers est le traducteur du Livre XII (1556). Subsiste en outre au moins un exemplaire du Quatorzième Livre d'Amadis de Gaule, Chambéry: François Pomar, 1575, traduction d'A. Tyron revue par J. Gohory (après Anvers, 1574); disponible sur Gallica. Sur Amadis en France, voir Amadis. Livre I, M. Bideaux (éd.), Paris, Champion, 2006, Introduction, p. 7-157; L. Guillerm, Sujet de l'écriture et traduction autour de 1540, Paris, Aux amateurs de Livres, 1988; Les Amadis en France au Xv1 ${ }^{e}$ siècle, N. Cazauran (dir.), Cahiers V.-L. Saulnier, $\mathrm{n}^{\circ} 17,2000$.

293. Livres XXII-XXIV: Paris, G. Robinot, O. de Varennes et Cl. Rigaud, in- $8^{\circ}, 1615$, privilège du $1^{\text {er }}$ déc. 1614.

294. Mireille Huchon a consacré plusieurs études à Herberay, notamment " Amadis, "Parfaicte Idée de nostre langue françoise" ", dans Les Amadis en France, op. cit., p. 183-200; "Les clefs du château d'Apolidon ", dans Devis d'amitié, Mélanges Nicole Cazauran, Champion, 2002, p. 167-190; "Traduction, translation, exaltation et transmutation dans les Amadis », dans Camenae, $\mathrm{n}^{\circ}$ 3, 2007, p. 1-10; voir aussi " Le roman, histoire fabuleuse ", dans Le Roman français au XVI siècle, ou le renouveau d'un genre, M. Clément et P. Mounier (dir.), Strasbourg, Presses Universitaires de Strasbourg, 2005, plus particulièrement p. 51-60.

295. À Anvers (1560) et à Lyon (1567), on publie un Thresor avant de publier la collection. Sur les Thresors d'Amadis, voir L. Guillerm, op. cit. p. 78-90; V. Benhaïm, «Les Thresors d'Amadis », dans Les Amadis en France, op. cit., p. 157-181; M. Bideaux, Amadis. Livre I, op. cit., p. 53-54 et 137. Les éditions d'Amadis à Anvers et Lyon ne reproduisent pas l'intégralité des nombreuses pièces liminaires des premiers livres.

296. Huguetan a pris deux fois l'initiative d'augmenter le Thresor: selon V. Benhaïm, «Les Thresors d'Amadis ", art. cit., p. 165, "Les impressions successives reproduisent les passages retenus par l'édition Groulleau de 1559 puis ceux des augmentations données par Huguetan en 1571, Waesberghe en 1572 et Huguetan en 1582. » 
ailleurs et, au début du $\mathrm{XVII}^{\mathrm{e}}$ siècle, réimprimé à Lyon seulement (J. A. Huguetan, 1601 et 1606; Pierre Rigaud, 1605). Ce sont les liminaires des traductions lyonnaises tardives (Livres XV-XXI), et principalement ceux du traducteur Gabriel Chappuys en tant que témoins de l'évolution de la conception du roman $\mathrm{au} \mathrm{XVI}^{\mathrm{e}}$ siècle, qui nous occuperont ici. On les trouvera rassemblés infra dans les annexes.

Si Nicolas Herberay des Essarts a traduit huit livres d'Amadis, Gabriel Chappuys est responsable de sept autres pendant la période où il vécut à Lyon, soit plus du quart de la collection des vingt-quatre livres. Ce Lyonnais d'adoption était le neveu du poète Claude Chappuys, valet de chambre de François ${ }^{\mathrm{er}}$ et son bibliothécaire. Né probablement à Amboise avant 1550 (1546?), G. Chappuys arrive à Lyon peu après la mort de son oncle. Il a sans doute peu de ressources et est employé comme " prelecteur ${ }^{297}$ ». Michel Bideaux a rassemblé des éléments biographiques dans son édition des Facétieuses journées. Très actif à Lyon dans la période 1576-1583, G. Chappuys quitte définitivement Lyon pour Paris après la mort de Belleforest ( $1^{\mathrm{er}}$ janvier 1583) et finit par lui succéder. Il devient $\mathrm{d}$ 'abord historiographe et annaliste puis garde de la bibliothèque du roi et enfin secrétaire-interprète du roi. Ses lecteurs peuvent suivre sa carrière au fil des titres des ouvrages ${ }^{298}$. Il continue de traduire nombre de textes italiens, espagnols et latins au point de mériter le qualificatif de " galérien de la version ${ }^{299}$ » ou de «tâcheron des lettres ${ }^{300}$ ». L'essentiel de sa production signée est conservée; il laisse entendre qu'il est l'auteur de textes signés par d'autres à la fin de l'épître liminaire à Maximilian de Bethune, lorsqu'il affirme qu'il a toujours été stimulé à écrire "sans autre fruict que celuy qui [lui] en est demeuré en conscience, et mesmes ayant mis en lumiere plusieurs escrits, souz le nom et au profit d'autres pour leur faire plaisir ${ }^{301}$ ». Claude Longeon, qui relève que

297. En 1581, «maistre Gabriel Chappuys» est témoin d'un acte notarié impliquant les demoiselles Gimbre et Jehan Huguetan et est désigné comme "prelecteur en l'imprimerie » de Loys Cloquemin. Voir H. Baudrier, Bibliographie lyonnaise, I, 87 et XI, 334, cité par M. Bideaux dans « Le choix d'un traducteur: Gabriel Chappuys et la composition des Facétieuses journées ", dans La Nouvelle française à la Renaissance, L. Sozzi (dir.), Genève-Paris, Slatkine, 1981, p. 543-556, ici p. 543, note 1; ainsi que dans Facétieuses journées, Paris, Champion, 2003, p. 21. Voir aussi L. Berthé de Besaucèle, Études sur l'évolution des théories littéraires en Italie au XvI siècle, suivie d'une notice sur G. Chappuys, traducteur français de Giraldi, Genève, Slatkine, 1969 [Paris, Picard, 1920] et P. Chavy, Traducteurs d'autrefois, Moyen Âge et Renaissance, I, Genève, Champion-Slatkine, 1988.

298. Il est « Annaliste, Translateur et Garde de la bibliothèque du roi » sur la page de titre de la Diane de G. de Montemaior (Tours, 1592); interprète en 1596 pour l'espagnol selon La Réception des écrits italiens en France à la Renaissance, Paris, Presses de la Sorbonne nouvelle, 2000, p. 96. En 1608, il signe encore «G. Chappuis S.I. du Roy » sa traduction des Cinquante meditations de toute l'histoire de la passion de nostre Seigneur, Du Latin de RPF Coster de la Compagnie de Jesus, Et mis en François pour la Consolation des Ames devotes, Paris, Robert Fouët (numérisé par Google Books). G. Chappuys meurt en 1612 ou 1613. César Oudin lui succèdera.

299. M. Bideaux, « Le choix d'un traducteur... », art. cit., p. 543.

300. Facétieuses journées, éd. cit., p. 8. M. Bideaux relève que de 1583 à 1585, Gabriel Chappuys fait paraître près de vingt ouvrages traduits pour la plupart de l'italien, de l'espagnol et du latin: «sa venue à Paris n'avait donc guère altéré sa (trop) grande fécondité ».

301. Cette déclaration est suivie d'une strophe ornée de comparaisons animalières: "J'ay faict quelques escrits: autres en ont l'honneur:/Ainsi pour soy l'Oyseau son nid rond ne maçonne,/ 
Chappuys était un ami d'Antoine du Verdier, l'a classé parmi ces « semi-docti » lyonnais aux personnalités trop faibles pour exercer une véritable influence ${ }^{302}$. Il est certain qu'il appartient aux minores. De sensibilité post-tridentine, il a contribué autant qu'il a pu à conforter l'autorité du roi et du pape.

Une fois à Paris, G. Chappuys dédie des traductions à Paul de Gondi, évêque de Paris; Charles de Lorraine, prince de Joinville; Louis Séguier, conseiller au Parlement; Philippe Desportes (pour sa traduction d'Equicola); au duc de Joyeuse. Vers la fin de sa vie, il a été chanoine. Il a traduit plusieurs auteurs spirituels (Jean d'Avila, Louis de Grenade), des sermons, des exposés doctrinaux, des méditations dévotes. Lié à des Jésuites, il était aussi probablement d'humeur ligueuse. Sa traduction des Leçons catholiques de Panigarola contient une réfutation de Calvin. Sa pensée politique, notamment celle de la Citadelle de la royauté, a été analysée récemment, notamment dans sa «sursacralisation royale $^{303}$ » qui aboutit paradoxalement à un déplacement des frontières entre le profane et le sacré qui prend appui sur les textes sacrés:

Or entre les hommes, les Princes reluisent comme le Soleil entre les autres estoilles: Ils sont l'ame du Royaume, par laquelle vivent et respirent tant de milliers d'hommes: et en quelque maniere, comme Dieu au monde: et pour ceste cause ils sont appellez Dieux, es sainctes lettres, es Pseaumes ${ }^{304}$.

Chappuys est aussi un adaptateur et un auteur. Guillaume Colletet note qu'il « composa peu de vers 305 ». M. Bideaux lui attribue quatorze des cent nouvelles des Facétieuses journées (privilège du 11 avril 1584) qui sont une traduction du recueil de Sansovino. Ce recueil n'eut qu'un succès relatif ${ }^{306}$. Toutefois, certaines des traductions de G. Chappuys ont fait date par leur clarté. Ainsi

Et la Brebis pour soy ne porte sa toison:/ L'Abeille ainsi pour soy ne rend miel à foison,/ Ainsi pour soy, le Boeuf la terre ne sillonne. ", Citadelle de la royauté. Contre les efforts d'aucuns de ce temps, qui par Escrits captieux ont voulu l'oppugner, Paris, Guillaume Le Noir, 1604, «Epistre», f. A 7, Gallica [NUMM 73755.

302. C. Longeon, Une Province française à la Renaissance, La vie intellectuelle en Forez au $\mathrm{XVI}^{e}$ siècle, SaintÉtienne, Centre d'études foréziennes, 1975, p. 290. Chappuys dédie le 31 janvier 1583 la traduction de La Suite de Roland Furieux avec ses «sommaires allegoriques " (Lyon, B. Honorat) à Geneviève du Verdier, fille d'Antoine, qui meurt de la peste en 1586. Il lui suggère de demander à son père une explication des « allegories » de l'épopée. Voir C. Longeon, op. cit., p. 367.

303. P.-A. Mellet, Les Traités monarchomaques (1560-1600), Genève, Droz, 2007, voir notamment p. 339347 et p. 352-354. La «sursacralisation royale» (expression de D. Crouzet) répond au processus de désacralisation orchestré par les Monarchomaques; voir D. Crouzet, Les Guerriers de Dieu, Seyssel, Champ Vallon, 2005, p. 545-570. Rappelons que l'intérêt de Chappuys pour la science politique se marque dans son Estat, description et gouvernement des royaumes et républiques du monde tant anciennes que modernes, Paris, Guillaume Cavellat, 1585, et dans sa traduction de G. Botero, Raison et gouvernement d'estat en dix livres, Paris, Guillaume Chaudière, 1599.

304. Citadelle de la royauté, éd. cit., " Preface », f. B, passage cité par P.-A. Mellet, op. cit., p. 382.

305. Vies des poètes tourangeaux, L. Locey, M. Locey et J. L. Pallister (éd.), Seattle-Tübingen, Papers on French Seventeenth Century Literature, 1989, p. 107. Citons les Figures de la Bible declarees par stances à Lyon, Barthelemi Honorati, 1582. Chappuys traduisit aussi L'Arioste.

306. Dans « Le choix d'un traducteur... ", art. cit., p. 555, M. Bideaux explique l'insuccès des Facétieuses journées par «le discrédit qui frappait alors la traduction littéraire et surtout la désaffection du public pour la nouvelle à l'italienne quand elle n'était pas parée des embellissements que les Histoires tragiques de Belleforest apportaient à l'œuvre de Bandello ». 
celle de Balthazar Castiglione, Le parfait courtisan du comte Baltasar Castillonois, les deux langues respondans par deux colonnes l'une à l'autre ${ }^{307}$ et celle de La Civile Conversation de Stefano Guazzo ${ }^{308}$. On remarque aussi sa recherche d'une certaine précision dans d'autres versions bilingues, ainsi Les colloques de Maturin Cordier divisez en quatre livres, traduicts de latin en françois l'un respondant à l'autre pour l'exercice des deux langues ${ }^{309}$. Ce n'est pas le cas de toutes ou, du moins - faut-il le rappeler? - elles furent rapidement dévaluées, et avec vigueur. Si l'auteur de la Bibliothèque des romans estime que G. Chappuys fut l'un des «Ecrivains les plus féconds et les plus estimés du seizième siècle », ses traductions lui paraissent "communément exactes mais sans aucune élégance » si bien qu'évoquant L'Histoire des amours extrêmes d'un Chevalier de Séville dit Luceman [Luzman], et de la belle Demoiselle Arbolea, il assure qu'elle paraîtra « assez intéressante, lorsque nous l'aurons dégagée du style ennuyeux et même assommant du Sieur Chappuis ${ }^{310} »$.

Reste que Chappuys est un « passeur » de textes importants et pas seulement dans le milieu lyonnais. Il est un continuateur assez avisé, quoique tardif, de la collection des Amadis mais n'est pas le seul: il eut des concurrents à Paris, Anvers et Lyon ${ }^{311}$. Tous insèrent leurs volumes en respectant la stabilité du titre, composé d'un ordinal, du nom d'Amadis de Gaule - même s'il ne s'agit plus que de ses descendants - et de la mention quasi constante d'une traduction de l'espagnol $^{312}$. Cette stabilité pendant soixante-quinze ans masque des difficultés de plusieurs types qu'il n'y a pas lieu d'énumérer ici. Retenons seulement que la numérotation française de la collection ne correspond pas toujours à celle de la collection espagnole, et que les conflits vécus par Amadis et tant $d^{\prime}$ autres chevaliers trouvent en quelque sorte une image dans les conflits ou la simple concurrence entre les gens du livre (libraires, imprimeurs, traducteurs).

307. Lyon, Loys Cloquemin, 1580. Cette traduction a été réélaborée dans B. Castiglione, Le Livre du Courtisan, A. Pons (éd.), Paris, G. Lebovici, 1987; voir p. XxXVII-XXXVIII. G. Chappuys a revu sa traduction: Paris, N. Bonfons, 1585, Gallica [NUMM-75479; Paris, A. L'Angelier, 1592.

308. Lyon, Jean Beraud, 1579 et 1580; pour cette dernière, Gallica [NUMM-108560.

309. Lyon, Cloquemin et Michel, 1576; réimpression à Paris, Hierosme de Marnef, 1586 et J. Libert, 1638. Sur ce texte, voir B. Colombat, La Grammaire latine en France à la Renaissance et à l'âge classique, Grenoble, ELLUG, 1999, p. 61-63; selon lui, « cette version bilingue doit permettre des progrès parallèles dans chacune des deux langues ». Les Mathur. Cordierii Colloquiorum scholasticorum Libri quatuor datent de 1564.

310. Bibliothèque universelle des romans, Paris, mai 1777, p. 103, et p. 101 pour la citation précédente. Les Estranges avantures de Jerome de Contreras (Lyon, B. Rigaud, 1580) sont republiées à Paris, N. Bonfons, 1587.

311. Livre Xv, traduit par A. Tyron, Anvers et Paris, 1577. Livre xvI, traduit par N. de Montreux, Paris, 1577. Livre XIX, traduit par Jacques Charlot, Lyon, Loys Cloquemin, 1581 (voir Annexe IV). Livre xx, traduit par Jean Boyron, Lyon, Guichard Jullieron, 1582.

312. Même et surtout s'il s'agit pour lui de traductions de continuations italiennes (il en va de même pour les Livres XXII-XXIV traduits de l'allemand à Paris en 1615). Chappuys mentionne toutefois des « aureilles Italiennes » dans l'épître du Livre XVI. Jacques Charlot évoque les « livres traittans de l'histoire d'Amadis de Gaule » lus à Rome mais prétend s'être ensuite procuré les livres espagnols (voir Annexe IV). 
Pourquoi et comment lire les Amadis selon Chappuys? Comment comprendre cet intérêt pour une collection qui connaît déjà une « disgrâce » analysée par Michel Simonin ${ }^{313}$ ? Le traducteur s'en explique dans des épîtres liminaires le plus souvent datées et adressées à quelques personnalités lyonnaises étudiées par Evelyne Berriot-Savadore ${ }^{314}$. Les liminaires parisiens ont été étudiés notamment par Sergio Cappello, Jean Lecointe, Rosanna Gorris et Michel Stanesco ${ }^{315}$.

\section{Des « dédications » pour que le livre « ne coure extravagant et incongneu »}

Si on les lit à la suite, ces épîtres liminaires lyonnaises d'Amadis paraissent - il faut l'avouer - un peu répétitives ou anodines mais elles esquissent cependant une défense du roman, voire une apologie, à un moment où le genre est toujours décrié. On fait l'hypothèse que ces épîtres ne relèvent pas d'une pure et simple stratégie personnelle d'auto-promotion. Certes, Chappuys ne manque pas de faire l'éloge de chaque dédicataire choisi et se montre en quête d'appuis lyonnais. Il évoque des envieux (Livre xv) et ses veilles (Livre XVI); il remercie Dieu d'être " venu à fin ». Son libraire proclame avoir rémunéré son traducteur ${ }^{316}$.

Chappuys cherche évidemment à étendre le lectorat des Amadis, ce dont lui a su gré le libraire, mais son intention n'est pas seulement commerciale. À partir du Livre XVI, il fait allusion à un dédicataire précédent, reliant ainsi les personnalités choisies dans une chaîne de lecteurs amis ${ }^{317}$. Parfois encore, il se plaît à rappeler que les premiers volumes de la collection étaient adressés à des rois et des seigneurs. Sa technique publicitaire va même au-delà. En tête de la traduction du Troisieme Livre de Primaleon, il relie ce roman aux Amadis et

313. M. Simonin, « La disgrâce d'Amadis », Studi Francesi, n 48, 1984, p. 1-35, repris dans L'Encre et la Lumière, Genève, Droz, 2004, p. 189-234.

314. Les Femmes dans la société française de la Renaissance, Genève, Droz, 1977, p. 377-381.

315. J. Lecointe, «Théorie du récit, aux marges de l'épopée et du roman dans les paratextes des Amadis au XVI ${ }^{\mathrm{e}}$ siècle en France », dans Du Roman courtois au roman baroque, E. Bury et F. Mora (dir.), Paris, Belles Lettres, 2004, p. 367-381. Cette étude met en lumière la notion d'accident et étudie le rôle de la fortune en lien avec l'épopée antique. S. Cappello, "I prologhi del Premier Livre d'Amadis de Gaule (1540) ", dans Filologia Moderna, Sezione Italiana e Romanza, 11, I, Pise, Pacini Editore, p. 25-41, et "Il Discours sur les livres d'Amadis de Gaule de Michel Sevin (1548) ", dans Il Romanzo nella Francia del Rinascimento, Fasano, Schena, 1996, p. 207-224. S. Cappello rapproche les liminaires des Amadis de ceux du Roland Furieux. R. Gorris, "Pour une lecture stéganographique des Amadis de Jacques Gohory ", dans Les Amadis en France au XvI siècle, op. cit., p. 127-156. M. Stanesco, "Premières théories du roman. Les folles amours des paladins errants », dans Poétique, n 70, 1987, p. 167180. Sur le liminaire de Mathurin Béhu (Livre v) et celui de Michel Sevin (Livre VIII), voir aussi U. Langer, Penser les formes du plaisir littéraire, Paris, Classiques Garnier, 2009, p. 76-79.

316. Épître de Jean Béraud en tête du Livre XIX (voir Annexe II): «j'ay fait traduire avec grans frais ». Toutes les épîtres sont désormais citées par le numéro d'ordre du Livre et reproduites en annexe.

317. L'épître du Livre xvı à Charles Capon fait mention de Madame Mandelot, dédicataire du Livre Xv ; celle du Livre XVII au Comte de Tillières à de "semblables » dédicataires précédents; celle du Livre XVIII à « ceux, ausquels j'ay dedié les trois livres precedens ». 
le dédicataire du Livre III de Primaleon à celui du Livre II, tout naturellement puisqu'ils sont cousins, et justifie le principe même d'une dédicace:

Toutesfois pour n'estre le premier qui ait consacré ces recreatifves (et en quelques endroits utiles et notables) traductions à personnages d'estoffe et de marque tels que vous, voire à Roys, Princes et grans seigneurs, comme l'on peut voir és dedications des premiers Amadis et plusieurs autres de tel suject, je me suis adressé à vous, d'autant plus hardiment que j'ay sceu la lecture du second avoir esté aggreable à monsieur vostre susdit cousin, joint que, luy ayant voué le precedent, qu'il a receu de si bonne volonté, il sembloit plus que raisonnable de vous presenter cestuy cy, tant à fin qu'il ne coure extravagant et incongneu, que pour declarer la bonne volonté que j'ay à vostre noble maison $[\ldots]^{318}$.

L'argument de l'ancienne dédicace royale d'Amadis est exploité d'autant plus facilement qu'elle était sous les yeux des lecteurs lyonnais. Les éditions lyonnaises d'Amadis ont en effet sélectionné quelques liminaires parisiens ${ }^{319}$. Ainsi Le Second Livre d'Amadis de Gaule (Benoist Rigaud, 1577) reproduit le douzain «Au Roy " ( Deux ans et plus Amadis m'a tenu ») et Le Cinquieme Livre (François Didier, 1577), l'importante épître liminaire «Au treschrestien Roy de France, François premier de ce nom ${ }^{320}$ ». Le fait qu'Herberay des Essarts ait été en mesure de dédier au roi lui-même tel livre d'Amadis devient, sous la plume de Chappuys, à la fois un élément de l'éloge de la personnalité lyonnaise choisie et un argument de légitimation de la collection entière des Amadis, même augmentée. Chappuys rend hommage à Herberay et s'affirme en continuateur.

L'éloge du dédicataire s'appuie classiquement sur l'éloge du nom, parfois de la lignée et surtout de la vertu ou des vertus du dédicataire. Ainsi lorsque G. Chappuys s'adresse L. Robertet, il dit «s'emparer de vostre haut nom »; dans l'épître à Ch. Capon, le livre personnifié « a ce bien de porter vostre nom dessus le front »; il paraît « sous l'appuy de vostre nom excellent et vertueux ». La mention est amplifiée pour le comte de Tillières au Livre XVII: «je me suis advisé d'honnorer mon livre du tiltre de vostre tres-haut et tres-noble nom ».

318. L'épitre est datée de 1579. Chappuys a donc traduit Primaléon dans les années d'Amadis.

319. Les éditeurs lyonnais abrègent parfois les titres des chapitres dans la Table. Dans celle du Livre v, les titres sont ainsi incomplets de leur fin et la coupure est signalée par « etc. ».

320. On se souvient que, dans cette épître, Herberay identifie des personnes royales aux héros du roman espagnol: "Sire, au retour des guerres d'Artois et Luxembourg, poursuyvant la Cronique d'Amadis, comme il vous a pleu me commander. Il m'a semblé que ce qui est escrit du Roy Perion et sa posterité, n'est autre chose que la figure de vous, et de messeigneurs vos enfants. ", Lyon, François Didier, 1577, f. a 2. E. Pasquier déclara qu'Herberay des Essarts voulut « représenter sous un Perion de Gaule, ce qui estoit de la vraye courtizanie ", dans Recherches de la France, M. M Fontaine et F. Roudaut (éd.), Champion, 2006, livre vIII, 5, p. 669. Le Livre v a été récemment édité: Garci Rodríguez de Montalvo, Le Cinqiesme Livre d'Amadis de Gaule, V. Duché (éd.) avec la collab. de J.-C. Arnould, Paris, Classiques Garnier, 2009. 


\section{«Maintenant j'ay cet heur que de semer en terroir heureux et fertile » (Livre XVI)}

L'histoire de la lignée d'Amadis est adressée à des familles lyonnaises. Une vertu « de pere en fils » est notée dans la dédicace à Ch. Capon; Chappuys évoque le père de Jan Anthoine Gros «qui laisse de soy lignée ne degenerant de sa vertu » (Livre XVIII) et la «noble maison » de Sainct Jouere (Livre XXI). Autour des dédicataires, surgissent ainsi des familles de lecteurs, d'honnêtes compagnies, des lignées sans reproche également pourvues des qualités du dédicataire principal. Ces qualités qui caractérisent le bon lecteur sont parfois énumérées dans la première dédicace, celle à Leonor Robertet au liminaire du Livre Xv: "vostre divin esprit, sens et jugement ». Ce public virtuel et idéalisé trouvera une honnête récréation dans ces traductions. Ni le libraire Béraud ni G. Chappuys ne nient qu'il ne s'agit que d'une récréation comme le fut le Premier Livre pour son dédicataire, Charles, le deuxième fils de François I $^{\text {er321. }}$.

L'évocation des vertus morales du dédicataire prend sens dans l'éloge des personnages de la fiction et l'éloge du roman lui-même, c'est-à-dire l'éloge de l'histoire racontée. Chappuys énumère dans la même phrase - mais pas le même ordre - les qualités des personnages fictifs et celles - réelles - qu'il voit en Nicolas Spina:

[...] et puis à qui pouvoys-je mieux adresser ce livre, qui descrit les vertuz et perfections des grands, illustres, amoureux, loyaux, genereux et gentils, qu'à vous qui estes l'exemple d'amitié, loyauté, magnanimité, vertu, generosité et gentilesse, et qui avez de la grandeur beaucoup?

L'éloge des héros s'appuie sur trois séries d'éléments: la valeur morale, l'exemple des vertus guerrières et celui de l'amour honnête. À Leonor de Robert et, épouse de François de Mandelot, il écrit que le Livre Xv délivre « un honneste discours là où il est parlé d'amour » dans une phrase qui loue les deux héros, don Silves et son épouse Pantasilee. Au Livre XvI, il précise à Ch. Capon que l'amour est « honneste et non lascif ». Rien de neuf: J. Gohory dans le liminaire du Livre XIII notait que les «traicts d'amour [...] y sont couverts d'honnesteté ${ }^{322}$ ». Certes, ce sujet n'est pas un «vray sujet » (J. Béraud, Annexe II $^{323}$. Si la fiction ne peut prétendre à la vérité de l'histoire, on peut du moins louer son invention. C'est le «sujet » même qui est défendu avec des

321. « Prologue du translateur »: " pour vous donner quelque fois dequoy recréer vostre gentil esprit, lorsqu'il sera ennuyé de lire choses plus haultes et ardues " et " pour le passetemps et plaisir ", M. Bideaux, Amadis. Livre I, op. cit., p. 167. V. Krause, Idle Pursuits: Literature and oisiveté in the French Renaissance, Newark, University of Delaware Press, 2003, p. 124 (y est notée la contrainte de lire). Amadis a aussi servi de recréation à Chappuys occupé par des travaux plus graves (Livre XVI).

322. Anvers, Guillaume Guzman, 1571, f. * 2 vº $^{\circ}$ Dédicace à Catherine de Clermont comtesse de Retz. V. Benhaïm évoque la censure de certains passages.

323. Sur les fictions qui sont toutes des « sujets caducs ", voir M.-L. Demonet « Les mondes possibles des romans renaissants ", dans Le Roman au xvie siècle..., op. cit., p. 126 sq, et F. Cornilliat, Sujet caduc, noble sujet, Genève, Droz, 2009. 
arguments moraux dès le Livre xv: " l'invention de ce livre tiré des Annales de Constantinople surpasse à mon avis le sujet de tous les precedens ». Le Livre XV posait du reste un problème puisqu'il ne suivait pas le Livre XIV traduit par Antoine Tyron, comme nous en prévient l'anonyme "Avertissement » qui suit l'épître de G. Chappuys (voir Annexe II) et aide le lecteur à se repérer ${ }^{324}$. Dans l'épître à Guillaume Gella, l'éloge du sujet d'Amadis prend la forme d'une énumération:

Je peux soustenir toutesfois que ce sujet n'est si bas que chacun ne puisse remarquer, avec un grand plaisir de beaux discours, l'exemple de toute honnesteté, force, prudence, vertu, loyauté et grande magnanimité: la liberalité tant recommandable aux grands y est remarquee: la beauté naifvement depainte, la justice representee au vif, et la vangeance des mesfaits et outrages que l'on fait aux pauvres affligez amplement descrite, de maniere qu'en tels escrits qui semblent, pour la pluspart fabuleux, se peut recueillir un grand fruit, touchant la maniere de vivre (Livre $x x)^{325}$.

Au-delà des lecteurs désignés qui constituent un public choisi, c'est à tous les lecteurs - y compris les Italiens - que Gabriel Chappuys garantit le plaisir des oreilles car l'invention est ingénieuse:

quand il n'y auroit que le plaisir et contentement que donne aux aureilles Italiennes et Françoises la gentile liaison d'une histoire tant ingenieusement inventee, il n'y a homme de bon jugement qui ne la juge digne d'estre leuë. (Livre XVI)

attendu que le livre est joyeux, ne traitant que de matiere pour donner plaisir, et contentement, à voz doctes aureilles ${ }^{326}$ (Livre XVII).

Le style d'interprétation de Chappuys n'a rien de commun avec celui de Jacques Gohory étudié récemment par Rosanna Gorris ${ }^{327}$, mais Chappuys suggère - sans trop de précision - que dans le Livre $x V$ « beaucoup de sens $y$ est comprins ${ }^{328} »$. Le traducteur fait allusion à Gohory dans le Livre xv sans le nommer: «le quatorzieme [Livre] precedent a esté dedié à une grande Dame

324. Sur cet exemple et sur la liaison entre la fin du Livre XVII et le suivant, voir C. de Buzon, « Amadis de Gaule en français: continuation romanesque, collection, compilation », communication au colloque organisé par l'ENS de Lyon et l'Université de Cambridge, Cambridge, 2008, à paraître dans French Studies, 2011.

325. Outre une valeur morale, les Amadis ont une valeur pratique illustrée par le Thésor des Amadis. Sur ce point, voir V. Benhaïm, "Les Thresors d'Amadis », art. cit., p. 169-173 et ici la p. 173, qui note le passage d'un lectorat adulte à la « jeune noblesse » dans le Thrésor, Lyon, J. A. Huguetan, 1582.

326. Même argument à Marguerite de Mandelot en tête des Aventures de Luzman: "Lisez donc hardiment, car je suis certain que vous n'y trouverez aucune chose qui offense vos aureilles [...] ». L'épître est datée de 1580, en pleine période de traduction des Amadis.

327. R. Gorris, « Pour une lecture stéganographique... », art. cit., p. 127-156.

328. Il se garde d'imiter Gohory mais suggère que l'histoire des Amadis est transposable dans le monde à divers niveaux et en diverses occasions: elle est donc utile. Gohory, au Livre XIII, évoque un " suc et moelle de doctrine » (op. cit., Anvers, 1571,f. *3) et rappelle, avant de nommer Charles IX, que François I ${ }^{\mathrm{er}}$ affirmait «par sa royalle eloquence, que sous l'escorce de ces joyeuses narrations, y gisoit de bonnes instructions moralles pour la noblesse en exaltant les vertueux faits et blasmant les vicieux, en recommandant tousjours l'adoration et reverence de Dieu, la defense du bon droit $[\ldots] »\left(\right.$ f. * $\left.3 \mathrm{v}^{\circ}\right)$. 
[Henriette de Clèves, duchesse de Nevers] pour le plaisir qu'elle prenait à la lecture des livres bien inventez. »

L'essentiel est que ce nouvel Amadis prenne place parmi les «bons livres» ou les « livres bien inventez » (Livre Xv); sa matière est « pour donner plaisir » et il a un " gentil subject » (Livre XVII). Chappuys parvient ainsi à lisser en quelque sorte le raccord, à raccrocher sa série italienne non seulement aux premiers et prestigieux volumes espagnols (et aux Italiens) mais aussi au Livre XIV traduit par Gohory. La dédicace du Livre XVIII est l'occasion de louer tous les livres, de provoquer une remémoration mais aussi une attente des livres à venir:

Vous recevrez donc, s'il vous plaist ce petit livre d'aussy bon œil que ont fait ceux, ausquels j'ay dedié les trois livres precedens m'asseurant que s'il vous plaist en avoir la lecture, vous y trouverez grande delectation, comme à la verité l'histoire qui y est descrite, et mesmes en tous les precedens et en ceux qui viendront apres, a esté inventee pour delecter, mais avec tant de beaux traits, et une infinité de divers accidens et occurrences qu'il est impossible qu'avec le plaisir et la delectation, l'on en tire un grand proffit, comme vous experimenterez, moyennant la grace de Dieu [...]. (Livre XVIII)

G. Chappuys veille au lien avec les travaux de ses prédécesseurs et préserve la fiction d'une continuité de l'univers amadisien sous le nom d'Amadis. Est-ce une continuité à défaut d'unité? Selon Mawy Bouchard, cette insistance serait à mettre en relation avec l'impératif d'unité requise dans les poétiques aristotéliciennes de Robortello, Scaliger et Castelvetro mais aussi aux reproches faits à ces romans sans fin, bons pour des Sisyphes (traducteurs ou lecteurs). Ajoutons que la définition de la poésie, «fondée selon Aristote en fiction » - et non sur l'emploi des vers - est alléguée par Aubert en tête de sa traduction ${ }^{329}$.

\section{«Pour la vertu, honneur et courtoisie qu'il enseigne »}

Il va de soi qu'on se trouve ici dans un cas de double énonciation: s'adressant à Leonor Robertet, à Charles Capon, au comte de Tillières, à Jean Anthoine Gros, à Guillaume Gella ou à Nicolas Spina, Chappuys vise constamment le lecteur à qui il propose un parcours d'interprétation, à qui il impose aussi sa conception d'une lecture profitable du roman, ce fruit qu'évoque le sonnet louant Chappuys, d'abord parmi les traducteurs (« ceux là qui le font vivre en France ») puis seul:

Goustez icy la divine eloquence

Le stile doux, la grace et la faconde

De chappus, et le fruict qui abonde

Souz ce discours (dont la perfection

Tire un chacun en admiration)

329. M. Bouchard, "Culture profane et tradition aulique», Avant le Roman. L'allégorie et l'émergence de la narration française au XVI siècle, Amsterdam-New York, Rodopi, "Faux titre", 2006, p. 220. Sur la question de l'unité, voir U. Langer, Penser les formes du plaisir littéraire, op. cit., p. 172-173. G. Aubert voit dans les romanciers des «imitateurs de la Poésie, fondée selon Aristote en fiction, contenant toutesfois des secrets d'erudition profonde ", et distingue mentir et dire mensonge, Livre XIII, op. cit., 1571, f. ** $\mathrm{v}^{\circ}$. 
Certes, le topos de l'humilité est bien récurrent dans les épîtres. Et le « petit present » de la traduction est offert bien que le sujet soit « bas »:

je ne pense m'estre trop abusé de vous avoir faict présent de ce gentil subject, attendant que ma plume s'esleve pour me suggerer quelque chose meilleure, dont quelque jour je vous puisse gratifier. (Livre XVII)

Je sçay bien que vous estes bien digne d'escrits plus graves et serieux, et que l'on me pourroit reprendre de vous dedier chose trop basse. (Livre $\mathrm{xxI}$ )

En tête du Livre xvII, le libraire Béraud s'excuse aussi topiquement d'une « chose » qui vient dans l'attente d'une «plus grande " (" attendant que je te face voir chose meilleure et plus grande, quand l'occasion se presentera »), mais c'est dans une épître où il argumente aussi en faveur de la supériorité de la fiction sur l'histoire pour éduquer les jeunes gens. Notons que le libraire Béraud reprend aussi un argument du « Discours » de G. Aubert au Livre $\mathrm{xII}^{330}$ et de sa «Preface» du Livre XIII sur la différence entre histoire et fiction ${ }^{331}$. La dimension éducative des Amadis était déjà annoncée dans l'épître à M. de Tillières, quoique courtoisement déniée à un dédicataire qui n'en a nul besoin:

[...] il est vray, monseigneur, que plusieurs pourroyent bien faire leur profit de ce livre, que je vous dedie, comme seroyent ceux qui sont apprentis en toute maniere de courtoisie, art des armes, et façon de bien parler: mais vous sçavez tout cela presque des vostre enfance, pource que des vostre enfance vous y avez esté instruit, nourry et entretenu.

Et dans l'épître suivante à Jean Anthoine Gros:

je me suis ingeré vous faire present de chose que j'ay pensé vous appartenir et estre convenable, de ce Dishuictiesme livre d'Amadis de Gaule, que j'ay traduit $\mathrm{d}^{\prime}$ Espaignol en nostre langue, pour la vertu, honneur et courtoisie qu'il enseigne, qui peut servir en mille occurrences et principallement quand on se trouve en notables compagnies, combien que ces choses là vous soient, je m'en asseure, propres et peculieres. (Livre XVIII)

On sait que les Thresors d'Amadis ont été utilisés comme des ouvrages scolaires par des précepteurs flamands entre 1580 et $1610^{332}$. Bien que « bas », le

330. Il s'agit de « contempler (comme en un theatre de tout l'univers) les variables changemens de la fortune, les remuemens des affaires du monde, l'inconstance des choses humaines, les hazards de la guerre, les trophées des princes vaincuz (choses qui se depaignent beaucoup mieux en une narration inventee qu'en une histoire veritable) ", Livre XII, Lyon, F. Didier, 1577, cité par J. Lecointe, "Lectures romanesques de Virgile à la Renaissance", Cahiers de l'Association internationale des études françaises, 2001, n 53, p. 191-212, ici p. 203.

331. «Par quoy cessent de mesdire ceux qui ne font cas que des histoires qu' on tient pour vrayes (dont il est aussi peu que Fenix). Car la plupart de ces croniqueurs sont plus à blasonner faux, en faisant profession notoire de verité, neantmoins inserent beaucoup de fauceté ou par hayne et envie des nations à eux contraires, ou par adulation des Princes vivans ou de leurs successeurs que ces Rommans recreatifs confessans clairement par leur tiltre leur invention fabuleuse ", Livre XIII, «Preface aux lecteurs ", f. ${ }^{* *} \mathrm{r}^{\circ}-\mathrm{v}^{\circ}$. Cité par M. Bouchard, Avant le Roman..., op. cit., p. 224.

332. V. Benhaïm, «Les Thresors d'Amadis », art. cit., p. 173 et p. 178-179. Pour suivre cette mode, Gervais Malot compose en 1581 un Trésor des Histoires tragiques. Pour la contenir, G. Sylvius invente en 1567 un Thrésor des Vies de Plutarque. 
roman diffuse des valeurs éternelles et universelles: justice, générosité, vengeance des outrages faits aux affligés comme on vient de le voir. L'univers amadisien est détaché de l'Espagne ou de l'Italie par Chappuys. Il n'est rattaché à la France que par le souvenir d'Herberay, des princes et de " l'ornement de la langue Françoise » (Livre XVI). En revanche, le sonnet qui loue Chappuys au Livre XVII ancre le roman à la "Gaule » en évoquant le "Gauloys Amadis » et « ceux là qui le font vivre en France » et ont ainsi rendu service à leur patrie. Notons que deux passages polémiques des liminaires visent probablement les réformés s'abstenant de lire des romans, « ceux qui les condamnent [...] et qui les blasment » par ouï-dire (Livre XvI) et les "ignorans, estourdis,/Qui detracte[nt] sans aucune apparence,/Des beaux discours du Gauloys Amadis » (Sonnet du Livre XVII) ${ }^{333}$.

Chappuys va un peu au-delà de la défense du sujet, de l'invention ingénieuse et de la subtile liaison, des traits et des accidents. Il tente de décrire plus techniquement Amadis même si son métalangage est réduit. Toujours pour Jean Anthoine Gros, il évoque la description, la propriété et l'élégance:

Mais comme nous ne sommes pas faschez d'ouir aucune fois bien et proprement deduire une chose que nous sçavons, ainsi paravanture ne dedaignerez vous de voir proprement et elegamment descris en ce livre maints actes vertueux, l'amour, la grace, la beauté, generosité, addresse, et courtoisie combien qu'en vous je retrouve veritablement tout cela.

En tête du Livre xx, on l'a vu, Chappuys évoque des situations et des qualités topiques de l'univers amadisien (méfaits, outrages, vengeances, libéralité, prudence, etc.), leurs descriptions (« naifvement », « au vif ») et les « beaux discours ». À relire le passage, on voit que l'éloge du texte en montre la variété, la cohérence et la dynamique (les actions de vengeance ont des causes qui sont les outrages), et enfin les vertus démontrées par des actes (« exemple de toute honnesteté, force, prudence »):

Je peux soustenir toutesfois que ce sujet n'est si bas que chacun ne puisse remarquer, avec un grand plaisir de beaux discours, l'exemple de toute honnesteté, force, prudence, vertu, loyauté et grande magnanimité: la liberalité tant recommandable aux grands y est remarquee: la beauté naifvement depainte, la justice representee au vif, et la vangeance des mesfaits et outrages que l'on fait aux pauvres affligez amplement descrite [...]. (Livre $x x$ )

En conclusion, revenons sur le sens des dédicaces qui évitent que le livre « ne coure extravagant et incongneu » et sur celui du livre où « beaucoup de sens y est comprins ». Gabriel Chappuys, conscient du «bénéfice de l'admirable imprimerie » (à N. Spina, Livre XXI) mais aussi de ses dangers dans une société $q^{\prime}$ 'on dit plus sécularisée ${ }^{334}$, éprouve peut-être fortement un sentiment

333. M. Simonin cite l'attaque ironique célèbre de Lescaldin, La Polémique protestante contre Ronsard, J. Pineaux (éd.), Paris, STFM, 1973, t. II, p. 277; voir L'Encre et la Lumière, op. cit., p. 234, n. 176.

334. V. Krause, "Serializing the French Amadis in the 1540's ", dans Charting Change in French Thought and Culture, M. Rothstein (dir.), Susquehanna University Press, 2006, p. 40-62. Cette étude rap- 
de responsabilité qui le conduit à prescrire cette lecture particulière capable de réduire ce caractère « extravagant et inconnu » de tout roman.

Dans cette même épître ultime, au moment donc de quitter les Amadis, il évoque ceux qui vont « vouer leurs œuvres à quelques excellens personnages, qu'ils cognoissent amateurs de la vertu, et des bonnes lettres, qui rendent les hommes vertueux ». Il ne se classe certainement pas parmi « ceux qui ne pensent qu'à leur particulier ». Enfin, il regrette que « les bonnes lettres semblent quasi estainctes ». Continuer les Amadis dans la nostalgie de l'époque de François ${ }^{\text {er }}$ (dont il fait l'éloge ailleurs) constituerait alors un moyen acceptable - tel livre est « joyeux » (au comte de Tillières, Livre XVII) - de procurer " plaisir et récréation » « aux amateurs de la vertu et des bonnes lettres ». Sans arguer davantage d'une valeur spirituelle, il présente les héros tardifs de la collection des Amadis comme des représentants de la justice, presque des héros chrétiens parfois animés par des missions collectives. On devine que lui plaisaient ces idéaux de croisade des rois catholiques, la réaffirmation de la fonction royale et surtout l'augmentation de la sphère d'influence de la chrétienté. Ce dernier point est par exemple illustré dans le chapitre «Comme les princes rencontrerent un chevalier Hebrieu en chemin: les propos qu'ils tindrent touchant la foy avec luy, un hermite et un Mahommetiste » et les chapitres suivants. Vingt-six pages plus loin, après des exposés doctrinaux, l'Hebrieu et le Mahommetiste sont baptisés et satisfaits ${ }^{335}$.

Conduisant la collection plus loin que ses prédécesseurs, veillant à la liaison entre les volumes, établissant enfin des liens entre ses lecteurs, Chappuys impose une unité idéologique forte qu'il faudrait confirmer en étudiant précisément le texte du roman et en le conférant avec les idéaux de la ContreRéforme mais aussi avec son assimilation de la culture profane. Bien avant deux évêques que nous lisons encore, Camus le romancier (né en 1584) et Huet auteur d'une apologie des romans (né en 1630), bien avant le succès de l'offensive jésuite au XVII ${ }^{\mathrm{e}}$ siècle, Chappuys témoigne de son admiration pour la prose romanesque. Le prosélytisme est absent des épîtres où il se borne à encourager la lecture profane d'un lectorat vertueux. Lyon n'est pas Genève où « à lire ou à vendre les Amadis on risque la prison ${ }^{336}$ ». Ce qu'il affirme du Livre XxI, «ce livre qui descrit les vertuz et perfections des grands, illustres, amoureux, loyaux, magnanimes, vertueux, genereux et gentils » conviendrait alors à tous les livres $\mathrm{d}^{\prime}$ Amadis sinon à tous les lecteurs. Les ennemis sont bien moins redoutables s'ils ne sont même pas nommés. Amadis peut devenir un opéra.

\section{Christine de BUZON \\ E. A. 1087, Université de Limoges}

proche Amadis des continuations de Rabelais et de la nouvelle pratique du prêt financier avec intérêt, et conclut à la sécularisation de la société.

335. Livre XV, chap. 44, p. 364 ; l'annonce du baptême se trouve p. 390.

336. M. Simonin, L'Encre et la Lumière, op. cit., p. 234, n. 176. 


\section{Annexes}

\section{Annexe I: épîtres liminaires des Amadis par G. Chappuys, Livres Xv-XXI}

1) Lyon, $1^{\mathrm{er}}$ février 1577: Le Quinziesme livre d'Amadis, Lyon: Benoist Rigaud, 1578 , p. 35.

À tresnoble et tresvertueuse Dame Leonor de Roberttet, femme et compagne de tresillustre Seigneur messire François de Mandelot, seigneur de Passy, Conseiller du Roy en son conseil privé, Capitaine de cinquante hommes d'armes des ordonnances de sa majesté, et son Lieutenant general és païs de Lyonnois, Forests et Beaujolois.

Madame, ceux là qui mettent la main à la plume à fin de publier leurs escrits et faire jouir le monde du fruit de leur labeur, ont volontiers accoustumé d'eux prevaloir et munir du nom de quelque grandeur de laquelle ils se couvrent pour obvier à la malignité de l'envie, laquelle fait tousjours la guerre aux doctes ouvriers en quelque chose que ce soit. Mais quant à moy, ce qui me fait emparer de vostre haut nom au commencement de ce livre, n'est pas pour [p. 4] la crainte que j'aye d'estre envié, en quoy je m'estimeroy bien heureux, pour ce que je sçay l'affection que vous avez à la lecture de tous bons livres François. Il est bien vray, madame, qu'au regard de votre divin esprit, sens et jugement, je n'ay d'aventure fait chose digne de vous estre presentee: mais la belle et plaisante invention du livre que j'ay translaté seulement suplera à ce defaut, et m'excuserez sur l'affection que j'ay de faire paroistre à un chacun que si le quatorzieme precedent a esté dedié à une grande Dame, pour le plaisir qu'elle prenoit à la lecture des livres bien inventez, je puis à bon droit vous consacrer ce quinziesme, sachant principalement que vous n'aymez pas moins à lire et que l'invention de ce livre tiré des Annales de Constantinople surpasse à mon avis le sujet de tous les precedens, d'autant que sous une fabuleuse [p. 5] histoire, beaucoup de sens y est comprins, comme vous pourrez voir. Car par les faits de don Silves de la Selve est proprement descrit l'exemple d'un bon, sage et secourable Prince et par son epouse Pantasilée, on peut parfaittement remarquer, l'honnesteté, grace, generosité et vertu des nobles et grandes Dames, avec un honneste discours là où il est parlé d'amour. Il vous plaira donc recevoir ce petit present de la main de celuy qui desire toute sa vie vous faire treshumble 
service, d'aussi bonne volonté qu'il prie Dieu, Madame, vous maintenir en sa sainte protection, et vous donner heureuse et longue vie. À Lyon ce premier jour de Fevrier 1577.

Vostre treshumble et affectionné serviteur, Gabriel Chappuys.

2) Lyon, 25 janvier 1578: Le Seiziesme livre d'Amadis de Gaule, Lyon, François Didier, 1578, f. a $2 \mathrm{r}^{\circ}$-a $3 \mathrm{v}^{\mathrm{o} 337}$.

À noble et vertueux seigneur Charles Capon, Seigneur d'Amberieu etc.

Monsieur, $\mathrm{j}$ 'ay apprins par le conseil et advertissement de Jamblic, que l'homme doit principalement avoir recours à la vertu, comme à son rampart, seureté et deffence, de peur qu'il soit exposé aux injures de fortune, et pour ceste cause sachant certainement comme de pere en fils, cete vertu a tousjours esté propre et peculiere à vostre noble maison, et comme en icelle vous avez tousjours esté nourry, instruit, et entretenu, encore qu'elle vous soit naturelle, je vous ay bien voulu adresser ce petit fruict d'aucunes miennes veilles, à fin que souz l'appuy de vostre nom [f. a $2 \mathrm{v}^{\circ}$ ] excellent et vertueux, il soit gousté d'un chacun à l'amiable, et que s'il n'est bien meur, ou qu'il y soit demeuré par avanture quelque aigreur, pour estre nouvellement cueilly, vostre douceur et debonnaireté la puisse corriger avec le temps, de maniere que le fruict que je vous presente soit trouvé de tous aussi savoureux, que celuy que l'annee passee je presentay à Madame de Mandelot, en la traduction que j'ay faite du precedent et quinziesme livre de ceste histoire: ce qui m'a meu de poursuivre et mettre la main à ce seiziesme, duquel Dieu mercy, je suis venu à fin, comme je croy, heureuse, puis qu'il a ce bien de porter vostre nom dessus le front, comme les premiers jusques au quinziesme le portent d'autres grands Seigneurs et grandes dames: ce qui m'excusera, si d'avanture quelqu'un vouloit maintenir que je vous ay dedié chose qui vous soit moins convenable, pour ce qu'elle est fabuleuse. Car il est certain que les grands ausquels ont esté consacrez tous [f. a $3 \mathrm{r}^{\circ}$ ] les autres, n'y eussent prins si grand goust et plaisir, si l'invention n'en eust esté subtile et digne d'estre leuë, sans une infinité de belles sentences servans d'instruction qui y sont semees, l'ornement de nostre langue Françoise, la justice qui s'y voit naifvement depeinte en la punition des geans, la generosité des Princes et Chevaliers en l'aide, et subvention des affligez, et de ceux qui sentent les fascheuses traverses de fortune, la pitié des dames et Damoiselles, et une infinité de beaux traits d'honneur et de courtoisie. Advisez donc, Monsieur, si ceux là qui pensent la lecture de ces livres estre inutiles, ne s'abusent pas en cet endroit: car outre le proffit que l'on en peut tirer, quand il n'y auroit que le plaisir et contentement que donne aux aureilles Italiennes et Françoises la

337. BnF [Y2 6228. Au verso: Extrait du privilège du Roy, 13 avril 1577 à Benoist Rigaud pour Le seiziesme livre d'Amadis de Gaule, traduict d'Italien en François par Gabriel Chappuis Tourangeau. 
gentile liaison d'une histoire tant ingenieusement inventee, il n'y a homme de bon jugement qui ne la juge digne d'estre leuë. Mais quoy? qui sont ceux là qui crient contre ces Romans? qui sont ceux [f. a $3 \mathrm{v}^{\circ}$ ] qui les condamnent? ceux qui ne mirent jamais le nez dedans, et qui les blasment souz ombre qu'ils ont entendu dire qu'ils ne parlent que d'amour. Je confesse qu'ils parlent d'amour, mais d'un amour honneste et non lascif, comme vous pourrez voir par experience en les lisant. Dieu m'a faict la grace de pouvoir mettre la main en choses plus serieuses que n'est ceste plaisante histoire ou autres Romans, et toutesfois je n'estime le temps que j'y ay vaqué entierement perdu et mal employé, tant pour le plaisir que j'en ay reçeu, apres mes estudes plus graves, que pour autant que maintenant j'ay cet heur que de semer en terroir heureux et fertile. Ce pendant, il vous plaira recevoir de bon cœur ce mien petit present avec une bonne devotion que j'ay de vous faire toute ma vie treshumble et tres-affectionné service. À Lyon ce 25. jour de janvier 1578.

Vostre treshumble serviteur, Gabriel Chappuys Tourangeau.

3) Le dixseptiesme livre d'Amadis de Gaule continuant à traiter des amours, gestes et faits heroiques des illustres et vertueux Princes Sferamond et Amadis d'Astre, Lyon, Estienne Michel, 1578, f. *2 $\mathrm{r}^{\circ}-* 3 \mathrm{r}^{\circ 338}$.

À Monseigneur le comte de Tillieres, de l'ancienne et tres-noble maison de Carrouges.

Monseigneur, sçachant comme volontiers vous favorisez ceux là qui s'efforcent mettre en avant quelque fruict de leurs labeurs, je me suis advisé d'honnorer mon livre du tiltre de vostre tres-haut et tres-noble nom, souz la confiance que j'ay qu'en ce temps de feste auquel est parlé de vostre bien heureux hymenee, il ne vous viendra pas mal à gré, attendu que le livre est joyeux, ne traittant que [f. ${ }^{*} 2 \mathrm{v}^{\circ}$ ] de matiere pour donner plaisir, et contentement, à voz doctes aureilles, puis que maintenant il est question de mettre toutes affaires serieuses souz le pied, combien que je desirerois maintenant avoir quelque meilleur ouvrage, pour vous presenter, qui fust digne de vous, c'est à dire, auquel il n'y eust que redire, il est vray, monseigneur, que plusieurs pourroyent bien faire leur proffit de ce livre, que je vous dedie, comme seroyent ceux qui sont apprentis en toute maniere de courtoisie, art des armes, et façon de bien parler: mais vous sçavez tout cela presque des vostre enfance, pource que des vostre enfance vous y avez este instruit, nourry et entretenu. Toutesfois considerant que les livres precedens qui traittent de semblable matiere, ont esté dediez les uns aux Rois et Princes: les autres à plusieurs grands personnages vos semblables, [f.

338. BnF [Y2 6229. Gallica: [NUMM 53117. Après la Table des chapitres: Privilège du 13 avril 1577 à B. Rigaud et mention « Ledict Rigaud a ceddé et remis ledict privilege à Estienne Michel marchand Libraire de Lyon ». À la fin du livre « Imprimé à Lyon par Estienne Brignol. 1578 ». 
*3] douez de grandes vertuz et accomplis en toutes les susdites parties, je ne pense m'estre trop abusé de vous avoir faict présent de ce gentil subject, attendant que ma plume s'esleve pour me suggerer quelque chose meilleure, dont quelque jour je vous puisse gratifier. Cependant je prieray Dieu le Createur, Monseigneur, vous donner en santé heureuse et longue vie, avec accomplissement de voz honnestes désirs.

Vostre tres-humble et tres-affectionné serviteur, Gabriel Chappuys.

[f. *3 $v^{\circ}$ ] DIZAIN.

Qui vouldra voir maintes lances briser,

Harnois froisser, escus tailler et fendre,

Qui voudra voir l'amant amour priser,

Et par amour les combats entreprendre,

Vienne Amadis visiter et entendre,

Que des Essars, par diligent ouvrage:

A retourné en son premier langage:

Et soit certain, qu'Espagne en cest affaire

Cognoistra bien, que France a l'avantage

Au bien parler autant comme au bien faire ${ }^{339}$.

\section{[f. *4] SONNET.}

Ou estes vous ignorans, estourdis,

Qui detractez sans aucune apparence,

Des beaux discours du Gauloys Amadis

Et de ceux là qui le font vivre en France:

Goustez icy la divine eloquence

Le stile doux, la grace et la faconde

De chappuis, et le fruict qui abonde

Souz ce discours (dont la perfection

Tire un chacun en admiration)

Alors je croy, si aveuglez vous n'estes,

Qu'il vous fera changer d'opinion,

Et retracter voz mesdits deshonnestes.

4) Lyon, $1^{\mathrm{er}}$ janvier 1578: Le dixhuitiesme livre d'Amadis de Gaule. Continuant les amours, gestes et faits heroiques des illustres et vertueux princes Sferamond et Amadis $d^{\prime}$ 'Astre, ensemble de plusieurs autres grands seigneurs y denommez par le plaisant et profitable discours d'une histoire en invention subtile et esmerveillable, autant et plus

339. Ce dizain, "Michel Le Clerc, seigneur de Maisons, Aux lecteurs », est, en 1540, la première pièce liminaire d'Amadis. Livre I, op. cit., p. 161. 
que les precedentes. Traduit d'Espagnol en François, Lyon, pour Loys Cloquemin, 1579 , p. $3-6^{340}$.

À noble et vertueux seigneur Jan Anthoine Gros, Sieur de S. Iovere, etc

Monsieur, l'obligation que j'avoy à feu monsieur vostre pere, à cause des plaisirs que j'ay receu de luy, me semble devoir estre à bon droit transferee à vous, qui estes non seulement heritier de ses biens et de ce qui [p. 4] luy estoit deu de temporel, mais aussi de ses rares et singulieres vertuz, et de ceste grande douceur et humanité, de laquelle il me sembloit user envers un chacun, de maniere que pour m'aquiter en partie du devoir auquel j'estoy tenu à l'endroit de luy, à juste cause, je m'adresse à vous, qui le representez si parfaitement en toutes choses, que l'on ne peut dire quasi qu'il ait encores payé le tribut de nature, $c^{\prime}$ est à dire, qu'il soit mort, comme à la verité l'homme vertueux ne semble pas mourir, qui laisse de soy lignee ne degenerant point de sa vertu, et gardant tousjours la bonne reputation d'iceluy. À quel propos cecy? C'est pour monstrer que comme en toutes choses vous [p. 5] me semblez tenir le gracieux et louable naturel de feu monsieur de S. Iovere (duquel j'estoy serviteur affectionné) tellement qu'il est du tout transformé en vous, et que vous estes tout un, aussy n'est mon affection aucunement changee et est toute une et telle envers vous, qu'elle estoit envers luy de maniere que voyant qu'il se perpetue en vous, perpetuant aussy mon service, je vous offre volontiers ce qui est en moy: joint que pere et fils sont relatifs, et que je ne puis avoir esté serviteur du pere, que je ne le sois maintenant du fils. À fin donc que je vous fasse paroistre combien je desire faire chose qui vous soit agreable, je me suis ingeré vous faire present de chose que [p. 6] j'ay pensé vous appartenir et estre convenable, de ce Dishuictiesme livre d'Amadis de Gaule, que j' ay traduit d'Espaignol en nostre langue, pour la vertu, honneur, et courtoisie qu'il enseigne, qui peut servir en mille occurrences et principallement quand on se trouve en notables compagnies, combien que ces choses là vous soient, je m'en asseure, propres et peculieres. Mais comme nous ne sommes pas faschez d'ouir aucune fois bien et proprement deduire une chose que nous sçavons, ainsi paravanture ne dedaignerez vous de voir proprement et elegamment descris en ce livre maints actes vertueux, l'amour, la grace, la beauté generosité, addresse, et courtoisie [p. 7] combien qu'en vous je retrouve veritablement tout cela. Vous recevrez donc, s'il vous plaist ce petit livre d'aussy bon œil que ont fait ceux, ausquels j'ay dedié les trois livres precedens m'asseurant que s'il vous plaist en avoir la lecture, vous y trouverez grande delectation, comme à la verité l'histoire qui y est descrite, et mesmes en tous les precedens et en ceux qui viendront apres, a esté inventee pour delecter, mais avec tant de beaux traits, et une infinité de divers accidens et occurrences

340 . BnF [Y²-6230. Au verso du titre: extrait du privilège en date du 24 octobre 1578 qui répète la mention « Traduit d'Espagnol en François ». 
qu'il est impossible qu'avec le plaisir et la delectation, l'on n'en tire un grand proffit, comme vous experimenterez, moyennant la grace de Dieu, lequel je supplie, [p. 8] Monsieur, vous maintenir perpetuellement en ses graces desirant avoir tousjours bonne part es vostres. À Lyon, ce premier jour de Janvier, 1579. Vostre treshumble et affectionné serviteur, Gabriel Chappuys Tourangeau.

5) G. Chappuys n'insère pas d'épître dans Le dixneuviesme livre d'Amadis de Gaule. Lyon, Jean Beraud, 1582. Voir ci-dessous l'épître du libraire, annexe II.

6) Lyon, 20 novembre 1580: Le vingtiesme et penultime livre d'Amadis de Gaule, Traittant des amours, gestes et faicts heroïques de plusieurs illustres et vertus princes de la race et souche dudict Amadis [...], Lyon: Loys Cloquemin, 1581, f. a 2 v- a $4 \mathrm{r}^{\circ 341}$.

À noble et vertueux seigneur Guillaume Gella, Salut et felicité.

Monsieur, le bruit de vostre vertu et gentilesse, m'a induit de vous faire present de ce petit livre, pour vous servir de recreation, m'asseurant qu'estant si bien nay que vous estes, vous ne l'aurez à mespris, souz ombre paravanture qu'il est moindre qu'il ne faut, pour cor- [f. a $2 \mathrm{v}^{\circ}$ ] respondre à vostre merite: car en cela, vous monstrerez vostre perfection et bonté, pource que les hommes bien advisez font aussi grand cas du petit present qui est faict de bon cœur, que de quelque grande chose que l'on sçauroit donner avec grande parade. Surce, il me souvient de l'exemple que lon raconte d'un de noz Rois, lequel ayant receu un present de quelques raves, par un simple homme des champs, les estima beaucoup, et recompensa le bon homme de beaucoup d'argent, ayant egard au bon zele et devotion d'iceluy: comme au contraire, il sceut bien cognoistre l'avarice et convoitise d'un certain courtisan, lequel prenant garde à la grande recompense que le [f. a 3] Roy avoit faite à ce bon homme, pour si peu de chose, s'advisa incontinent de luy faire present de quelque beau et gentil cheval, faisant argument qu'il emporteroit une recompense encores meilleure: mais le Roy cognoissant son desir, et ayant receu le cheval, fit apporter les raves du paisan, et les lui donna en recompense. Je peux soustenir toutesfois que ce sujet n'est si bas que chacun ne puisse remarquer, avec un grand plaisir de beaux discours, l'exemple de toute honnesteté, force, prudence, vertu, loyauté et grande magnanimité: la liberalité tant recommandable aux grands y est remarquee: la beauté naifvement depainte, la justice representee au vif, et la [f. a $3 \mathrm{v}^{\circ}$ ] vangeance des mesfaits et outrages que l'on fait aux pauvres affligez amplement

341. Colophon: Fin du vingtiesme et penultiesme livre d'Amadis de Gaule. Imprimé à Lyon Par Estienne Brignol. L'extrait du privilège (8 juillet 1580 ) figure après la Table des chapitres. Il est suivi de la mention «Achevé d'imprimer le vingtiesme Novembre, 1580 ». Disponible sur Gallica: [NUMM- 53092. La BnF conserve aussi un exemplaire de 1580. 
descrite, de maniere qu'en tels escrits qui semblent, pour la pluspart fabuleux, se peut recueillir un grand fruit, touchant la maniere de vivre. J'ay dedié les livres precedens à plusieurs de vostre étoffe, entre autres, l'un à Monsieur de Sainct Jouere, vostre beau frere, pour la grande amitié et faveur que j'ay autresfois receu de feu monsieur Sainct Jouere, son pere, duquel la memoire est recommandable: en consideration dequoy, pour continuer tousjours ma bonne volonté en ses successeurs, et alliez, je me suis ingeré vous saluer de ce petit livre, que vous recevrez, s'il vous plaist, [f. a 4] d'aussi bon cœur, qu'il vous est presenté: et je prieray Dieu le Createur, Monsieur, vous donner, en santé, sa saincte grace. De Lyon, ce 20. jour de novembre 1580.

Vostre treshumble et affectionné serviteur, Chappuys.

7) Lyon, 20 février 1581, Le vingt uniesme et dernier livre d'Amadis de Gaule, Contenant la fin et mort d'iceluy, les merveilleux faicts d'armes et amours de plusieurs grands et notables Princes de son sang, et les divers et estranges effects d'un amour chaste et honneste: d'où en fin, l'on peut recueillir grand fruict, plaisir et contentement, Lyon, Loys Cloquemin, 1581, f. *2 $\mathrm{r}^{\circ}-* 3 \mathrm{v}^{\circ 342}$.

À noble et vertueux Seigneur Nicolas Spina, Salut et felicité.

Monsieur, comme la coustume soit quasi de tous ceux qui mettent la main à la plume, pour publier leurs escrits, et les mettre en lumiere, par le benefice de l'admirable imprimerie, de vouer leurs œuvres à quelques excellens personnages, qu'ils cognoissent amateurs de la vertu, et des bonnes lettres, [f. * $2 \mathrm{v}^{\circ}$ ] qui rendent les hommes vertueux, je vous ay voulu choisir pour un de ceux là (qui sont aujourd'huy rares en ce pays, où les bonnes lettres semblent quasi estainctes) à qui je puisse dedier ceste mienne traduction du vingtuniesme et dernier livre d'Amadis de Gaule, seulement pour vostre plaisir et recreation, aux heures qu'il vous plaira employer à la lecture d'iceluy. Je sçay bien que vous estes bien digne d'escrits plus graves et serieux, et que l'on me pourroit reprendre de vous dedier chose trop basse, toutesfois quand l'on considerera que les premiers livres de ceste plaisante histoire ont esté consacrez, aux Roys, princes et grands seigneurs, lesquels volontiers y prestoyent [f. ${ }^{*} 3 \mathrm{r}^{\circ}$ ] l'aureille, on cognoistra paravanture que je n'ay point failly de vous faire ce present: et puis à qui pouvoy-je mieux adresser ce livre, qui descrit les vertuz et perfections des grands, illustres, amoureux, loyaux, genereux et gentils, qu'à vous qui estes l'exemple d'amitié, loyauté, magnanimité, vertu, generosité et gentilesse,

342. Bandeaux typographiques aux initiales LC. Privilège du 8 juillet 1580 après la Table et avant le texte, n. p. Signé L'Ortin. Suivi de la mention «Achevé d'imprimer le vingtiesme de Febvrier, 1581. " Après le texte, p. 448: «Fin de l'histoire d'Amadis de Gaule, comprinse en vingt et un livres ». Suivi de la mention « Achevé d'imprimer le 20. de Febvrier 1581. » Gallica NUMM 53093. L'exemplaire de la Bnf [Rés. Y2 1406 suit ligne à ligne cette édition de L. Cloquemin mais avec un matériel typographique différent (selon la notice de la $\mathrm{BnF}$ ). 
et qui avez de la grandeur beaucoup? Mais je ne soucie pas de ce que l'on die, pourveu que je sache que mon petit labeur vous soit agreable, et que vous daigniez le favoriser, comme je $\mathrm{m}^{\prime}$ asseure, que considerant la bonne volonté de celuy qui le vous offre, vous le ferez, d'autant plus volontiers que je sçay bien que vous [f. ${ }^{*} 3 \mathrm{v}^{\circ}$ ] estes du tout eslongné de l'air de ceux qui ne sont naiz que pour eux mesmes, et qui ne pensent qu'à leur particulier, pource qu'il me semble et $\mathrm{m}^{\prime}$ asseure ainsi (bien que je ne vous aye pratiqué) que vous avez l'honneur en recommandation, et que vous surpassez encores le bruit qui court de voz vertuz. Il vous plaira donc recevoir cet œuvre en bonne part de celuy qui vous demeurera tousjours fort affectionné, là où il sçaura que vous y preniez goust et plaisir. Cependant il priera tousjours Dieu le createur pour vostre prosperité et santé. De Lyon ce vingtiesme jour de Fevrier, 1581.

Vostre treshumble et affectionné serviteur Gabriel Chappuys, Tourangeau.

\section{Annexe II: deux épîtres de libraires accompagnant des traductions de Chappuys}

1) «Avertissement" anonyme, Le Quinziesme livre d'Amadis, Lyon, Benoist Rigaud, 1578, p. 6.

Avertissement.

Amy lecteur, on t'avoit promis à la fin du quatorziesme precedent qu'il seroit traité au quinziesme, de Spheramonde et d'Amadis d'Astre, mais c'estoit pource que la continuation des faits de dom Silves que tu voirras icy, estoit incogneuë au translateur du livre susdit, laquelle depuis est venue en lumiere et a esté tirée des annales de Constantinople, $t^{\prime}$ asseurant bien que si elle manquoit ou estoit laissee derriere, l'histoire d'Amadis perdroit le plus beau et le plus naïf de sa grace et lumiere, comme il te sera facile à juger. Parquoy la matiere susdite de Spheramonde, d'Amadis d'Astre et autres excellens princes sera par nous remise au seiziesme ensuyvant, pour ne laisser icy la plus belle et plus notable partie de cet excellent Romant.

2) Épître non datée de Jean Béraud. Le Dixneufiesme livre d'Amadis de Gaule. Traittant des amours, gestes, et faicts heroïques, de plusieurs illustres et magnanimes princes nepveux d'Amadis de Gaule, et faisant mention de plusieurs autres grandz Seigneurs, par le plaisant et profitable discours d'une hystoire belle et agreable, sur toutes les precedentes. Traduit d'Espagnol en langue Francoyse, par Gabriel Chappuis Tourangeau. À Lyon, par Jean Beraud, 1582, p. *3 $\mathrm{r}^{\circ}{ }^{*} 4 \mathrm{v}^{\circ 343}$.

343. Le verso du titre est blanc. P. *2: extraict du privilege; p. ${ }^{*} 2 \mathrm{v}^{\circ}$ : fin du privilege "Donné à Paris le dernier jour de Janvier, l'an de grace mil cinq cens septante-neuf. » Signé De L'Estoille. 
Le Libraire au lecteur S.

Amy lecteur, plusieurs se sont destournez de la lecture des Romans, par ce que desireux (dient ils) de la verité, ils sont asseurez au contraire de ne trouver en tels escrits rien que fables et mensonges; prisans de maniere le nom d'Histoire, que lors qu'ils voyent quelque livre le porter en frontispice, ils le cherissent, et $l^{\prime}$ embrassent de tout point. En quoy certes tels, pour se monstrer ainsi zelateurs du vray, seroient fort louables, si la verité estoit [p. $\left.{ }^{*} 3 \mathrm{v}^{\circ}\right]$ tellement mariee à $l^{\prime}$ histoire, que l'histoire sans la verité ne peust subsister. Mais comme ce variable et monstrueux Protee se desguisoit en telle forme et figure qu'il vouloit, pareillement l'histoire se desvestant de sa propre qualité, qui est d'estre veritable, si elle veut estre vraye histoire, s'affuble le plus souvent de la faulseté, d'un Achille façonnant un Thersite, et d'un Thersite un Achille. Chose qui, comme j'estime, induisit jadis maints doctes et rares esprits à fantasier, et discourir à plaisir, voyans qu'il estoit mal aisé, voire diray-je, impossible, de dresser une juste histoire, et estimans que tels plaisans discours dressent mieux $l^{\prime}$ homme à gentillement escrire, que ne fait l'histoire qui nous amuse du tout au sens: et que les faux combats, et faulses victoires bravement descrites, peuvent aussi bien duire la [p. *4] jeunesse, que les faits d'armes les plus veritables. Telle façon de faire feust jadis receuë par Homere, Heliodore, Apulee, Virgile, et depuis par Arioste et beaucoup d'autres, et tellement practiquee par l'auteur de nos Amadis, mesmes en ce dixneufviesme volume, qu'en ta faveur j'ay fait traduire avec grans frais, où les valeureux et heroïques faits d'aucuns genereux et invincibles princes de la lignee d'Amadis, et autres, sont dictez de tant bonne grace, et si bravement poursuiviz, que le temps que tu mettras à le lire je m'asseure que tu le tiendras pour bien employé. Il contient aussi la fin et conclusion de la guerre que les payens meurent aux Chrestiens en l'empire de Grece: et le commencement du siege de Taurique en Perse, où les payens assemblerent des forces presque egales à celles que Xerxes [p. * $4 \mathrm{v}^{\circ}$ ] mena en Grece lorsqu'il la voulut envahir: où se voyent tant de finesse, tant de ruses belliques, tant de stratagemes, tant de vertueux et inimitables faits d'armes, que (comme l'on dit de la peinture qui feint de si pres le naturel, qu'il n'y manque que la parole) la fiction y est tant bien coulourée, qu'il est a pleindre, que l'auteur n'ait tourné son stil à quelque vray sujet. Je te presente donc cest ouvrage, te priant de recevoir de pareil cœur, que je desire te gratifier: attendant que je te face voir chose meilleure et plus grande, quand l'occasion se presentera. À Dieu.

Numérisation Google Books de l'exemplaire Munich, Bayerische Staatsbibliothek. BnF [Y²- 6231. Pour ce même Livre XIX, Loys Cloquemin publie la traduction de J. Charlot avec un privilège du 16 novembre 1580. Signé De Verton. L'épître de Charlot figure dans l'annexe IV. 


\section{Annexe III: mentions des Amadis dans d'autres épîtres liminaires de Chappuys}

1) Lyon, $1^{\mathrm{er}}$ mars 1579: Le Troisiesme livre de Primaleon de Grece, fils de Palmerin d'Olive, Empereur de Constantinople. Auquel les faits heroïques, mariages et merveilleuses amours d'iceluy, sont tant bien deduites et exprimees, que le lecteur outre le profit, n'en peut recueillir sinon plaisir et contentement. Traduit d'Espagnol en François. De nouveau corrigé et augmenté. Paris: Estienne Valet, 1587, f. a 2 rª $3 \mathrm{v}^{0344}$.

À noble et vertueux seigneur Cesar Panse salut et felicité.

Monsieur, pour autant que j'avois promis à la fin du second livre de Primaleon (Roman autant gentil et plaisant à lire apres les serieuses occupations qui vous detiennent, qu'autre qui se puisse trouver) mettre la main à ce troisiesme, je me suis acquité de ma promesse, et en suis venu à bout, pour le faire voir en public, sous l'appuy et deffense de vostre nom, ny plus ny moins que j'ay fait du second, sous le nom honnorable de monsieur vostre cousin I. Pense, personnage autant gracieux et recongnoissant comme il est d'un cœur vrayment noble et genereux, auquel pour ceste raison là, je demeureray obligé toute ma vie, sous esperance de luy dedier quelque jour, et à vous aussi œuvres paravanture plus graves et plus convenables à voz qualitez. Toutesfois pour n'estre le premier qui ait consacré ces recreatifves (et en quelques endroits utiles et notables) traductions à personnages d'estoffe et de marque tels que vous, voire à Roys, Princes et grans seigneurs, comme l'on peut voir és dedications des [f. a $2 \mathrm{v}^{\circ}$ ] premiers Amadis et plusieurs autres de tel suject, je me suis adressé à vous, d'autant plus hardiment que j'ay sceu la lecture du second avoir esté aggreable à monsieur vostre susdit cousin, joint que, luy ayant voué le precedent, qu'il a receu de si bonne volonté, il sembloit plus que raisonnable de vous presenter cestuy cy, tant à fin qu'il ne coure extravagant et incongneu, que pour declarer la bonne volonté que j'ay à vostre noble maison, attendant la commodité de vous monstrer plus ample tesmoignage d'affection et service, en vous dediant, comme j'ay desja dict dessus, quelque ouvrage de plus grand poix, comme à la verité, je sçay bien que vous le meritez. Car quant à cestuy cy, je vous confesse qu'il tend plutost au plaisir, qu'à autre chose plus serieuse, n'estoit qu'il m'est advis que l'on doit faire cas de la subtile invention de ceste fabuleuse histoire, laquelle m'a fait entreprendre de la tourner en nostre vulgaire, estant delibéré, Dieu aydant, la poursuivre jusques à la fin, comme j'ay quasi fait des livres $\mathrm{d}^{\prime}$ Amadis de Gaule qui tendent à une mesme fin, enseignans neantmoins tous les traits de courtoisie et generosité, par la deduction d'une histoire qui plaist et

344. Munich, Staatsbibliothek, numérisé par Google Books. 
laquelle veu ce que dessus, on me confessera aussi estre aucunement profitable à ceux qui ne sont ny courtois, ny genereux, ny recognoissans. Mais on me dira, puis que vous avez ces parties de courtoisie, generosité, et recongnoissance que je me devois addresser à [f. a 3] autre qui peust faire son profit de la lecture de ce livre quand je l'eusse fait, peut estre ce que c'eust esté en vain, pource que ou il me le falloit dedier à quelqu'un qui eust l'esprit encores tendre et propre à ployer, comme le jeune osier, ou à autre (quoiqu'ignorant d'humanité et courtoisie) qui fust desja ferme et constant en son rustique naturel: mais de dedier ce livre au premier, les poincts amoureux (que la nature enseigne assez) m'en ont empeché: et si je l'eusse voué à autre, ignorant, rude et mal traitable, je vous prie quel gré est ce qu'il m'en eust peu sçavoir? il n'eust peu gouster le plaisir ny le profit qui se peut tirer de ce livre, et par consequent eussé-je esté bien tost condamné comme celuy qui ne s'adonne qu'à traduire livres de peu d'effect et de consequence jusques là, que j'estime et juge qu'il eust peu dire que je ne sers en mes traductions qu'à perdre temps et penser donner plaisir. Mes labeurs peuvent tesmoigner si mon intention a tousjours esté telle et si tousjours j'ay pensé à perdre temps, combien que veritablement je confesse que ce seroit perdre temps d'honnorer telles manieres de gens des premices de mes ouvrages, pource que (encores qu'ils soient petits) ils en seroient du tout indignes. Voila pourquoy je m'adresse à vous, comme à celuy qui n'estes ignorant des poincts susdits, et qui pour ceste cause en sçaurez equitablement juger, priant Dieu le createur. [f. a $3 \mathrm{v}^{\circ}$ ]

Monsieur vous avoir en sa saincte garde et protection avec accroissement de tous biens. De Lyon ce premier jour de mars 1579.

Vostre treshumble et affectionné serviteur, Gabriel Chappuys.

2) Lyon, 20 janvier 1580. Avantures amoureuses de Luzman Chevalier espagnol, et d'Arbolea sa maistresse. Où sont representez diverses Amours, des perseverances, rencontres et avantures, qui leur arriverent en dix ans, que ces amoureux errerent pas le monde. Mis d'Espagnol en François, Rouen: Theodore Reinsart, 1598, f. A 2-A 3 $\mathrm{v}^{\circ 345}$.

À tres-noble et vertueuse damoiselle, Marguerite de Mandelot, fille de tres-vertueux et tres-magnanime, Monseigneur Messire François de Mandelot, Seigneur de Passi, Chevalier de l'ordre du Roy, Capitaine de cinquante hommes d'armes de ses ordonnances, Gouverneur et Lieutenant general, pour sa Maiesté, és pays du Lyonnois, Forests et Beaujolois etc.

Madame, sçachant bien que ma traduction du quinziesme livre d'Amadis de Gaule, que j'ay dedié à Madame vostre mere, n'a esté d'elle mal receuë, selon 
l'humanité et bonté de laquelle elle est plaine, j'ay pensé que si je vous dediois quelque mien labeur, vous le prendriez d'aussi bonne part qu'elle a fait, entant que vous tenez (comme damoiselle sage, vertueuse et bien apprinse) des bonnes parties qui sont en elle: car ainsi comme vous participez des nobles traicts et lineamens de sa personne, vous participez aussi des nobles vertus de son esprit. Qui fait que je me suis volontiers ingéré de vous adresser ce petit œuvre, tant pour monstrer l'affection que j'ay à vostre noble et illustre maison, que pource que vous pouvez voir en iceluy exemple des plus honnestes et chastes amours d'un gentil-homme et damoiselle, que l'on vid jamais, estans accompagnees d'avantures tant estranges et esmerveillables, qu'il n'y a personne les lisant, qui n'en reçoive un tresgrand plaisir et recreation. Lisez donc hardiment, car je suis certain que vous n'y trouverez aucune chose qui offense vos aureilles [...] sans l'intention que j'avois de vous le presenter, je n'y eusse jamais mis la main, et oncques (ce croy-je) n'eussay-je trouvé aucun moyen d'en venir à bout, à cause de quelques autres miennes occupations. [...] »

Annexe IV : épître liminaire du Livre XIX traduit par Jacques Charlot, Lyon, Loys Cloquemin, 1582, f. * $2 \mathrm{r}^{\circ}-4 \mathrm{r}^{\circ 346}$.

Au lecteur, Jacques Charlot Champenois, salut.

Comme j'estois n'a pas longtemps à Romme à la suite d'un grand et vertueux Cardinal François mon bon seigneur et maistre, je m'employois aucunes fois à la lecture tant des livres Latins que vulgaires: entre autres je prenois par intervalles grand plaisir, à lire les livres Italiens, lesquels comme je vinsse à manier, un jour [f. * $2 \mathrm{v}^{\circ}$ ] me tomberent entre les mains certains livres traittans de l'histoire d'Amadis de Gaule, ausquels je prins une si grande recreation que dés lors je proposay traduire en nostre langue ceux qui n'avoyent encores esté veus en icelle, à sçavoir le Dixneuf, Vingt et Vingtuniesme: pource que je n'avois veu lesdicts livres traduicts que jusques au Dixhuictiesme de la traduction fort agreable d'un certain Tourangeau. Et pour ce faire la commodité s'y presenta beaucoup meilleure que je n'eusse pensé, d'autant que je trouvay moyen de recouvrer d'un gentilhomme Hespagnol, les livres dudict Amadis de Gaule, en langue Hespagnolle (laquelle j'avois aprinse, pour avoir demouré long temps avec mondict Seigneur, en la court d'Hespagne), [f. 3] et pour ceste cause je me mis hardiment à encommencer mon project, et de fait je vins à bout du Dixneufième, estant deliberé de poursuivre les autres jusques à la fin. Mais comme mondict Seigneur m'eust envoyé à la court de France, passant par la ville de Lyon je sceu par Loys Cloquemin marchand libraire en ladicte ville, que

346. BnF [NUMM 53091. Privilège du 16 novembre 1580. Signé De Verton. Dernière page: «Achevé d'imprimer le deuxiesme Janvier, 1581. Par Estienne Brignol ». 
le susdict Tourangeau estoit apres à traduire lesdicts Vingt et Vingt et uniesme livres dudict Amadis de Gaule, et qu'il avoit presque tout achevé. Parquoy, je m'arrestay tout court en cest endroit et proposay ne poursuivre mon labeur encommencé, sachant que ce que je voulois faire, estoit desja faict par la main $\mathrm{d}^{\prime}$ un, duquel la traduction estoit desja tant bien receue, et au [f. ${ }^{*} 3 \mathrm{v}^{\circ}$ ] quel à ceste cause je n'eusse voulu faire tort, tant à raison de la suffisance de son stile, que pource que paravanture mon labeur n'eust esté tant favorisé que le sien. Et de faict estant à Lyon j'avois deliberé faire de ce Dixneufiesme un sacrifice à Vulcan, mais je fus prié par ledict Cloquemin de le luy laisser: ce que je fis ne pensant pas qu'il deust venir en lumiere, comme il est venu par une juste occasion que ledict libraire mien amy m'a donnee à entendre. Qui a esté cause, amy lecteur, de faire que je luy ay, quasi contre mon gré, envoyé ceste lettre, pour te l'adresser, et te supplier par icelle de m'avoir pour excusé, si d'avanture je n'ay eu la grace en tout et par tout, de contenter tes aureilles, $t^{\prime}$ advisant, que je n'ay pas eu le loisir (pource que [f. *4] l'affaire ainsi que l'on me mandoit, requeroit toute diligence) d'eslire quelque Seigneur, à qui je peusse vouer mon œuvre (que je n'avois encores bien limé, ne pensant pas que jamais il deust voir le jour) à fin d'estre garanty et appuyé contre les injures des langues mesdisantes, qui ne servent qu'à detracter des honnestes labeurs d'autruy, combien que je ne pense avoir si bien faict, que paravanture un autre n'ait mieux faict que moy. Toutesfois, comme dict bien Ciceron, il n'est mal seant d'avoir la seconde place, quand on ne peut venir et attaindre à la premiere. À Dieu, amy Lecteur, te desirant tousjours la grace celeste, et perpetuelle felicité. Escrit d'Esparnay en Champagne, le huictiesme jour de Novembre, 1580. 
